\title{
Melting line of polymeric nitrogen
}

\author{
L.N. Yakub \\ Thermophysics Department, Odessa State Academy of Refrigeration, 1/3 Dvoryanskaya Str., Odessa 65082, Ukraine \\ E-mail: unive@icn.od.ua
}

Received December 5, 2012

\begin{abstract}
We made an attempt to predict location of the melting line of polymeric nitrogen using two equations for Helmholtz free energy: proposed earlier for cubic gauche-structure and developed recently for liquid polymerized nitrogen. The $P-T$ relation, orthobaric densities and latent heat of melting were determined using a standard double tangent construction. The estimated melting temperature decreases with increasing pressure, alike the temperature of molecular-nonmolecular transition in solid. We discuss the possibility of a triple point (solidmolecular fluid-polymeric fluid) at 80 GPa and observed maximum of melting temperature of nitrogen.

PACS: $05.70 . \mathrm{Ce}$ Thermodynamic functions and equations of state;

61.66.Bi Elemental solids;

61.50.Ah Theory of crystal structure, crystal symmetry; calculations and modeling;

64.70.dj Melting of specific substances.
\end{abstract}

Keywords: solid nitrogen, polymerization, phase transition, equation of stare.

\section{Introduction}

Recent studies of solid nitrogen at high pressures [1] revealed existence of its new crystalline phases. Polymorphism is typical for molecular cryocrystals but the specific feature of nitrogen is that some high-pressure phases of solid nitrogen are nonmolecular [2].

Experimental confirmation of the polymerization in solid [3] and liquid nitrogen [4] allow theorists and experimentalists to discuss the issue of a new configuration of the phase diagram of solid nitrogen at high pressures.

The calculated $P-T$ line of the molecular-to-polymeric transition in solid nitrogen [5,6] reveals essential dependence on the structure of polymeric phase. Phase transitions with rearranging of chemical bonds are typical not only for nitrogen, but for many simple molecular condensed systems build from molecules with multiple chemical bonds.

The possibility of molecular-to-polymer transition in liquid nitrogen was discussed in relation to the discovery of the temperature drop and increased conductivity of the nitrogen fluid behind the reflected shock wave (shock cooling) discovered by Nellis et al. [7].

$A b$ initio simulations of Boates and Bonev [8] reveal that dense liquid nitrogen may also have complex structure, similar to that found in the solid nitrogen. The transition from the molecular to the atomic structure can be interpreted as a break triple chemical bond in $\mathrm{N}_{2}$ and formation of a network of ordinary chemical bonds con- necting each $\mathrm{N}$ atom with three its nearest neighbors in the polymeric structure.

Thus, the general idea, which may explain the phenomenon of polymerization in the liquid and solid phases, is the same. This allows using the equation of state (EOS) of the solid phase and liquid phase polymer nitrogen line to predict the melting crystalline polymeric nitrogen into polymeric liquid.

In this work we use a new EOS for highly compressed polymer nitrogen liquid which was developed recently and calibrated on results of $a b$ initio simulations and applied to the prediction of the liquid-liquid transition in highly compressed nitrogen [9]. Using two equations of state: for polymeric solid and polymeric liquid nitrogen one can calculate the location of the melting line on $P-T$ diagram, and densities of coexisting phases.

\section{EOS for polymeric nitrogen solid}

We applied the modified Mie-Grüneisen model and EOS for anharmonic polymeric solid proposed in our work [10]. This EOS describes the thermodynamic properties of solid nitrogen in a wide range of parameters of state in cubic gauche (cg)-polymeric phase, and predicts the negative thermal expansion and significant deviations of heat capacity from the Dulong-Petit law. This EOS was used earlier in prediction of the molecular-to-polymer phase transition in solid nitrogen [6]. 
The Helmholtz free energy of an anharmonic solid was written [10] as a sum:

$$
F_{\text {poly }}=F_{\text {poly }}^{(h)}+F_{\text {poly }}^{(\text {anh) }},
$$

where $F_{\text {poly }}^{(h)}$ is the quasi-harmonic contribution, represented by the modified Mie-Grüneisen model, and $F_{\text {poly }}^{(\text {anh })}$ is an anharmonic correction:

$$
F_{\text {poly }}^{(h)}(T, \rho)=U_{\text {poly }}^{(0)}(\rho)+3 R T D_{F}\left(x_{D}\right) .
$$

Here $U_{\text {poly }}^{(0)}$ is the energy of static lattice, and $D_{F}\left(x_{D}\right)$ is the Debye function:

$$
D_{F}\left(x_{D}\right)=\frac{3}{x_{D}^{3}} \int_{0}^{x_{D}} \ln \left(1-\mathrm{e}^{-x}\right) x^{2} d x ; \quad x_{D}=\frac{\theta_{D}}{T} .
$$

Both thermal and caloric EOS are easily obtainable from Eq. (1) by differentiation with respect to density and temperature:

$$
\begin{gathered}
P(T, \rho)=\rho^{2} \frac{\partial}{\partial \rho} F(T, \rho) \\
E(T, n)=-k_{B} T^{2} \frac{\partial}{\partial T} \beta F(T, n) .
\end{gathered}
$$

Here $\beta=\left(k_{B} T\right)^{-1}$.

As it was shown in Ref. 10, the anharmonic contributions to the heat capacity as well as to the thermal expansion and isothermal compressibility are important.

The anharmonic contribution includes anharmonic corrections $A_{1}(\rho)$ and $A_{2}(\rho)$ :

$$
\frac{F_{\text {poly }}^{(\text {anh })}}{R T}=-\frac{A_{1}(\rho)}{2} T-\frac{A_{2}(\rho)}{6} T^{2},
$$

and $A_{1}(\rho)$ and $A_{2}(\rho)$ were found in Ref. 10 using deviations of the heat capacity from the Dulong-Petit law, deduced from Monte Carlo data [11] for cubic gauche (cg)-solid nitrogen:

$$
\begin{gathered}
A_{1}(\rho)=-0.004918 \rho^{*}\left(\rho^{*}-1.0468\right)\left(\rho^{*}-0.8481\right), \\
A_{2}(\rho)=4.03 \cdot 10^{4} \rho^{*}\left(\rho^{*}-0.9666\right)\left(\rho^{*}-0.8763\right), \\
\rho^{*}=\rho V_{0}, V_{0}=7 \mathrm{~cm}^{3} / \mathrm{mol} .
\end{gathered}
$$

The quasi-harmonic thermal Grüneisen parameter, $\gamma=$ $=\partial \ln \theta_{D} / \partial \ln \rho$, was determined by extracting the anharmonic corrections, calculated according to Eqs. (4) and (5) from the Monte Carlo data [11] on pressure and energy. Surprisingly, it was found to be almost independent of temperature and decreasing nearly linear with the increasing density. Equations for $\theta_{D}$ and density-dependent Grüneisen parameter:

$$
\gamma=\gamma_{0}\left(1-\frac{\rho}{\rho_{0}}\right)
$$

includes three constants: $\gamma_{0}, \rho_{0}$ and $\theta_{D}^{(0)}=\theta_{D}\left(\rho_{0}\right)$, where $\rho_{0}$ is the density corresponding within quasiharmonic approximation to $\gamma=0$. All the constants were determined from Monte Carlo data [11]: $\gamma_{0}=30.5, \rho_{0}=$ $=1 / 7 \mathrm{~cm}^{3} / \mathrm{mol}$, and $\theta_{D}=200 \mathrm{~K}$.

The linear decrease of the thermal quasi-harmonic Grüneisen parameter with density [10] gives the possibility for a simple modification of the Mie-Grüneisen model. Expression for the quasi-harmonic contribution to the Helmholtz free energy as a function of temperature $T$ and volume $V$ remains the same as in the original Mie-Grüneisen model.

Applying the standard thermodynamic relations one can obtain expressions for quasi-harmonic contributions to all thermodynamic functions. Equations for the energy and heat capacity remain the same as in the original Mie-Grüneisen model, except for the new density dependence of the Debye temperature.

All the EOS parameters used in this work were adopted from Ref. 10 except the static lattice energy $U_{\text {poly }}^{(0)}$, which was shifted by $\Delta E_{0}$ - the difference in energies between static molecular and static cg-lattices. The value of $\Delta E_{0}$ parameter is important in calculation of the phase equilibrium. Zhang et al. [12] refer to $\Delta E_{0}$ value of $1 \mathrm{eV} / \mathrm{atom}$. In our calculations we adopted the value $\Delta E_{0}=0.97 \mathrm{eV} /$ atom obtained by Mailhiot et al. [13].

\section{EOS for liquid polymeric phase}

EOS of polymerizing fluid nitrogen was written [9] as an expression for Helmholtz free energy $F$ of a mixture of $\mathrm{N}_{2}$ molecules, dimers $\mathrm{N}_{4}$ and all other possible polymers $\mathrm{N}_{2 k}$ is as follows:

$$
F=F_{\text {poly }}^{(\mathrm{id})}+\Delta F^{(H D)}\left(\eta_{1}\right)+\alpha \Delta F_{\text {poly }}\left(\eta_{L}\right) .
$$

Here $n_{1}$ is numerical density (concentration) of molecular component $\left(\mathrm{N}_{2}\right), \eta_{1}=\pi n d^{3} / 3$ is "molecular" and $\eta_{L}=$ $=\pi n d_{L}^{3} / 3$ is "polymeric" packing fraction, $n$ is the molar density of nitrogen, $\alpha$ is the degree of polymerization: $\alpha=1-n_{1} / n$.

The general expression for ideal-gas part for Helmholtz free energy can be written as:

$$
F_{\text {poly }}^{(\mathrm{id})}=k T V \sum_{k=1}^{\infty} n_{k} \ln \left(\frac{n_{k}}{Q_{k}(T)}\right),
$$

where $Q_{k}(T)$ are partition functions of $k$ th component: $k=1\left(\mathrm{~N}_{2}\right), k=2\left(\mathrm{~N}_{4}\right)$, etc. The explicit expression of the

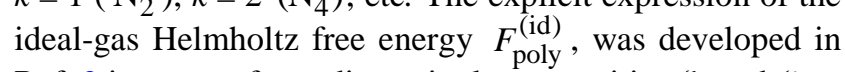
Ref. 9 in terms of two dimensionless quantities $\xi$ and $\zeta$ :

$$
\begin{aligned}
& \beta F_{\text {poly }}^{(\mathrm{id})}=\frac{\zeta}{\xi(1-\zeta)^{2}} \times \\
& \times\left[\varepsilon(1-2 \zeta)-\zeta+\frac{\varepsilon}{\zeta}(1-\zeta)^{2} \ln (1-\zeta)-(1+\varepsilon \zeta) \ln \left(\frac{Q_{1}(T) \xi}{\zeta}\right)\right] .
\end{aligned}
$$

Auxiliary variables $\xi$ and $\zeta$ are defined [9] as follows: 


$$
\begin{aligned}
& \zeta=\frac{2 \xi}{1+2 \xi+\sqrt{1+4 \xi(1+\varepsilon)}} \\
& \xi=\frac{n K_{2}(T)}{1+\varepsilon / 2} \exp \left(\frac{6 \eta_{L}}{1-\eta_{L}}\right) .
\end{aligned}
$$

The polymerization degree $\alpha$ can be expressed in terms of $\xi$ and $\zeta$ as:

$$
\alpha=1-\zeta / \xi \text {. }
$$

Effect of nonideality $\Delta F^{(H D)}\left(\eta_{1}\right)$ was included by the hard-dumbbell contribution [14].

The contribution of the strong attraction between atoms in polymerized fluid leads to formation of a network of interatomic single bonds and is accounted by the last term in Eq. (10). It is a function of the "polymeric" packing fraction $\eta_{L}=\pi n d_{L}^{3} / 3$ proposed in Ref. 15:

$$
\beta \Delta F_{\text {poly }}\left(\eta_{L}\right)=-\frac{6 \eta_{L}}{1-\eta_{L}} .
$$

The above Eqs. (10)-(16) completely define the EOS for the polymeric liquid.

EOS for polymeric liquid nitrogen was calibrated on $a b$ initio simulation data [8] and applied to prediction of fluid-fluid transition in strongly compressed nitrogen [9]. The $\Delta E_{0}$ value was fitted to reproduce the melting pressure near the triple point at $1500 \mathrm{~K}$ [1].

\section{Results and conclusions}

The pressure-temperature relation, orthobaric volumes and latent heat of melting were determined using a standard double tangent construction. Orthobaric volumes of the coexisting liquid and solid phases of polymeric nitrogen were obtained as abscissa of the point of contact of the common tangent to the curves of the free energy Eq. (2) and Eq. (10) and the equilibrium pressure of melting was defined as the slope of this tangent. The results are presented and compared with experimental data in Figs. 1 and 2.

It should be noted that EOS for polymeric liquid [9] was calibrated on $a b$ initio data [8] ranging from 2000 to $5000 \mathrm{~K}$ and hence the solution appear to be possible only within the limited range of temperatures and pressures. At temperatures above $\sim 1750 \mathrm{~K}$ the predicted melting line crosses the (extrapolated) liquid-liquid phase separation line and therefore no above common tangent was found. At temperatures below $1500 \mathrm{~K}$ the extrapolation of EOS for liquid phase become, in our opinion, too far.

In Fig. 1 we present the predicted temperature-pressure relation on the melting curve of polymeric nitrogen. Predicted melting temperature is compared here with experimental data and the molecular-to-plastic transition line predicted earlier [6]. Note that the estimated melting temperature decreases with increasing pressure, alike the temperature of molecular-nonmolecular transition in solid.

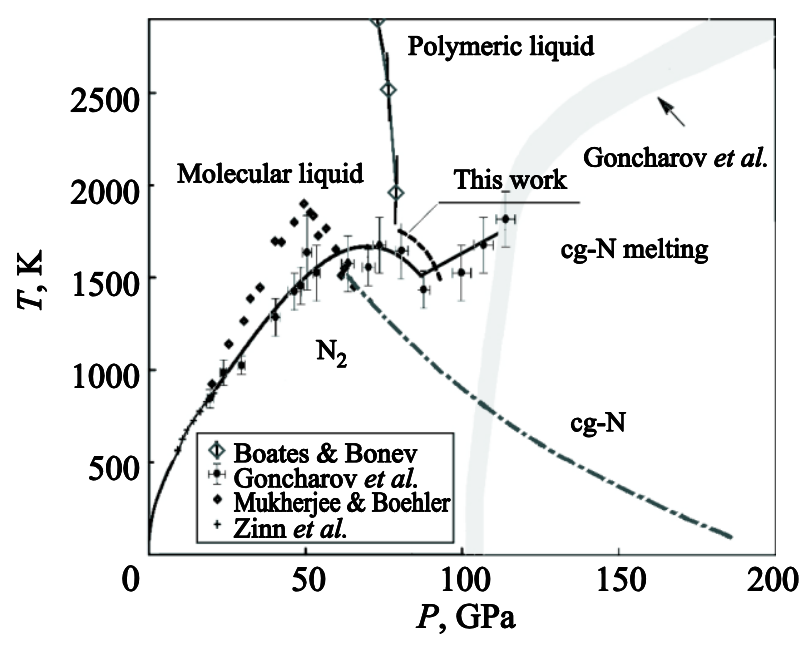

Fig. 1. Temperature-pressure dependence of the nitrogen melting line. Predicted melting temperature (dashed line) in comparison with experiments [1,17,18], ab initio [8] data, and molecular-topolymeric solid-solid transition (dash-dotted line), predicted earlier [6]. Light gray area: phase boundary of cg-N estimated in Ref. 1.

The predicted pressure dependence of the orthobaric volumes of the solid and liquid polymeric nitrogen on the melting curve are shown in Fig. 2 together with the only available experimental data Eremets et al. [16] for the molecular (circles and squares) and polymeric nitrogen (diamonds) at room temperature. This comparison gives an idea of the volume change during melting of polymeric cg-phase in that limited range of pressures where the calculations have been carried out. There is a reasonable quantitative agreement with the room-temperature data of Eremets

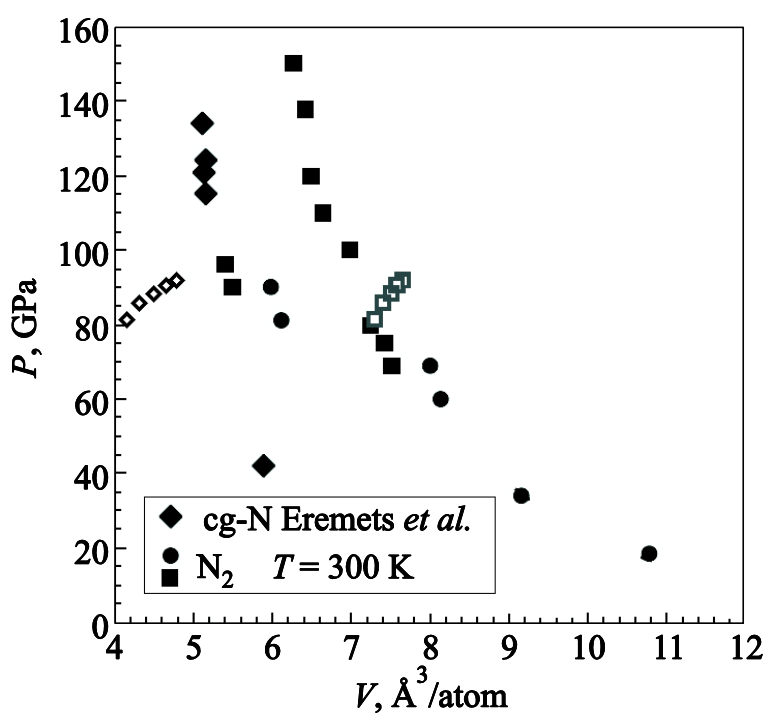

Fig. 2. Predicted pressure-volume dependence of the solid (open diamonds) and liquid (open squares) polymeric nitrogen on the melting line. Solid symbols represent experimental data of Eremets et al. [16] for molecular (solid circles and squares) and polymeric nitrogen (solid diamonds) at room temperature. 
et al. [17]. As one can see in Fig. 2, volumes of polymeric solid at high temperature are less than measured at room temperature. And the unusual increase of both volumes with pressure is not surprising. It due to negative slope of $P(T)$ relation and negative thermal expansion of polymeric nitrogen [11].

The estimated latent heat $L$ of cg-N melting is also negative and its absolute value increases with temperature. At $T=1700 \mathrm{~K}$ the value of latent heat reaches $-2.0 \mathrm{eV} / \mathrm{atom}$.

Recent $a b$ initio simulations [8] predict existence of at least a second triple point (solid-molecular fluid-polymeric fluid) at $P_{\mathrm{tr}} \sim 80 \mathrm{GPa}$ on the melting line of nitrogen. Our calculations support this prediction.

The predicted melting temperature decreases with the increasing pressure. It drops from $1750 \mathrm{~K}$ at $80 \mathrm{GPa}$ up to $1500 \mathrm{~K}$ at $95 \mathrm{GPa}$ (see Fig. 1). Such behavior is in line with the recent measurements of Goncharov et al. [1] who observed the maximum of the melting temperature.

1. A.F. Goncharov, J.C. Crowhurst, V.V. Struzhkin, and R.J. Hemley, Phys. Rev. Lett. 101, 095502 (2008).

2. Yanming Ma, A.R. Oganov, Zhenwei Li, Yu Xie, and J. Kotakoski, Phys. Rev. Lett. 102, 065501 (2009).

3. A.F. Goncharov, E. Gregoryanz, H.K. Mao, Z. Liu, and R.J. Hemley, Phys. Rev. Lett. 85, 1262 (2000).

4. W.J. Nellis, N.C. Holmes, A.C. Mitchell, and M. van Thiel, Phys. Rev. Lett. 53, 1661 (1984).
5. L.N. Yakub, Fiz. Nizk. Temp. 19, 531 (1993) [Low Temp. Phys. 19, 377 (1993)].

6. L.N. Yakub, Fiz. Nizk. Temp. 37, 543 (2011) [Low Temp. Phys. 37, 431 (2011)].

7. H.B. Radousky, W.J. Nellis, M. Ross, D.C. Hamilton, and A.C. Mitchell, Phys. Rev. Lett. 57, 2419 (1986).

8. B. Boates and S.A. Bonev, Phys. Rev. Lett. 102, 015701 (2009).

9. E.S. Yakub and L.N. Yakub, Fluid Phase Equil. In press. http://dx.doi.org/10.1016/j.fluid.2012.09.011.

10. L.N. Yakub, J. Low Temp. Phys. 139, 783 (2005).

11. L.N. Yakub, Fiz. Nizk. Temp. 29, 1032 (2003) [Low Temp. Phys. 29, 780 (2003)].

12. T. Zhang, S. Zhang, Q. Chen, and L.-M. Peng, Phys. Rev. B 73, 094105 (2006).

13. C. Mailhiot, L.H. Yang, and A.K. McMahan, Phys. Rev. B 46, 14419 (1992).

14. D.J. Tildesley and W.B. Street, Molec. Phys. 41, 85 (1980).

15. E.S. Yakub, Zh. Fiz. Khim. 87, 305 (1993).

16. M.I. Eremets, A.G. Gavriliuk, N.R. Serebryanaya, I.A. Trojan, D.A. Dzivenko, R. Boehler, H.-K. Mao, and R.J. Hemley, J. Chem. Phys. 121, 11296 (2004).

17. S. Zinn, D. Schiferl, and M.F. Nicol, J. Chem. Phys. 87, 1267 (1987).

18. G.D. Mukherjee and R. Boehler, Phys. Rev. Lett. 99, 225701 (2007). 